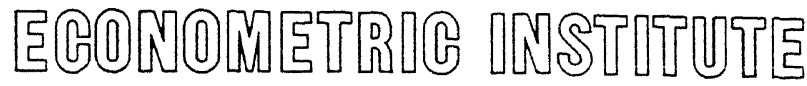

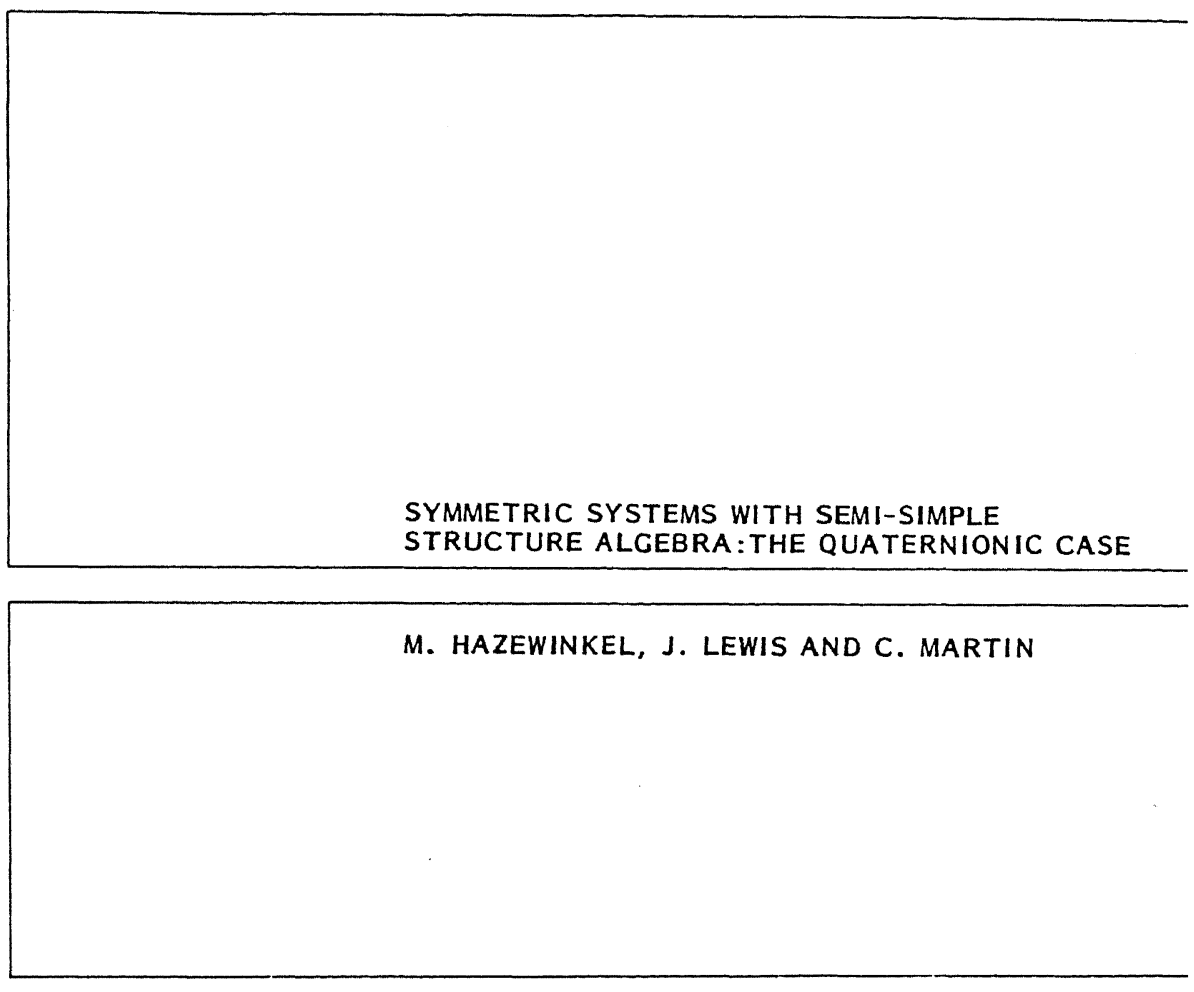

REPRINT SERIES no. 365

This article appeared in "Systems \& Control Letters", Vol. 3 (1983).

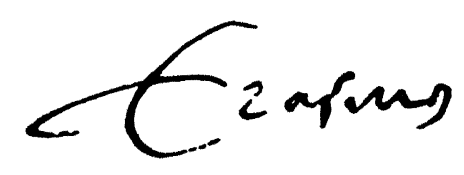

ERASMUS UNIVERSITY ROTTERDAM - P.O. BOX 1738 - 3000 DR ROTERDAM - THE NETHERLANDS 


\section{Symmetric systems with semi-simple structure algebra: The quaternionic case *}

\section{HAZEWINKEL}

Stichting Mathematisch Centrum. Kruisloan 413. $1098 \mathrm{SJ}$ Amsterdam. The Netherlands

\section{J. LEWIS **, C. MARTIN ***}

Dept. of Mathematics and Statistics. Case Institure of Technolog. Case Western Reserve Unicersit!: Cleceland. $\mathrm{OH} 44106$. USA

Received 13 March 1983

Revised 26 May 1983

The basic theory of linear systems over the quaternions is developed

Kẹnords: Symmetric systems. Quaternions. Semı-simple algebras.

\section{Introduction}

In [5] and [2] the concept of symmetric linear systems was introduced and the fundamental role of the structure algebra was established. We recall those concepts here for the benefit of the reader. Let $\leqslant$ be a class of linear systems where the dimensions of the state and input spaces are fixed and the $(A, B)$ satisfy some set of relations. The archetypical example is

$\mathcal{E}_{1}=\left\{\left(\begin{array}{cc}A & H \\ -H & A\end{array}\right) \cdot\left(\begin{array}{ll}B & 0 \\ 0 & B\end{array}\right)\right.$ :

$$
A, B, H \text { real matrices? }
$$

which arises in a variety of contexts and specifically in the modeling of twin-lift helicopters [6].

The structure algebra of the class $G$ is defined to be the algebra

- This work was partially performed while the first and third authors were at Erasmus U'niversits.

* Supported in part by NASA Grant No. NAG2-82

*.* Supported in part hy NASA Grants Nos. 2384 and NAG2-82. and DOE Contract $=$ DE-A01-80RAS236.

$$
\begin{gathered}
R(G)=\{(S . T): \text { for all }(A . B) \in \mathbb{E} \\
S A=A S . S B=B T\} .
\end{gathered}
$$

The importance of the structure algebra stems from the observation that because of commutatitively relations the state space and input space become $R(\sigma)$-modules and the maps $A$ and $B$ are module homomorphisms. Thus if the structure of $R(E)$-modules is known it is possible to reduce the structure of the maps $A$ and $B$.

In the case that the algebra $R(\mathbb{E})$ is semisimple. a great deal is known [2]. Houever in [2] it was shown that the symmetric systems with real semisimple structure algebra $R(\bar{\delta})$ could aluays be written as the direct sum of ordinary real systems. ordinary complex systems and 'ordinary' quaternionic systems. Unfortunately there is not a well established theory of linear svstems over the quaternions. The goal of this paper is to establish a minimal amount of material so that the theory of real. semisimple symmetric systems is somewhat complete.

\section{Linear algebra over the quaternions}

2.1. In the following we let $\boldsymbol{H}$ dencte the real division algebra of quaternions. Recall that a typical quaternion $h$ has the form

$h=a+b \mathrm{i}+c \mathrm{j}+d \mathrm{k}$

where 1. i. j. $\mathrm{k}$ form a basis for $\mathrm{H}$ as an $\mathbf{R}$-vector space. Multiplication in $\mathbf{H}$ is determined $h$ t the formulas

$\mathrm{i}^{2}=\mathrm{j}^{2}=\mathrm{k}^{2}=-1 . \quad \mathrm{ij}=\mathrm{k} . \quad \mathrm{jk}=\mathrm{i} . \quad \mathrm{ki}=\mathrm{j}$.

Also recall that $\boldsymbol{H}$ can be represented as the set of matrices of the form

$$
\left(\begin{array}{cccc}
a & b & c & d \\
-b & a & d & -c \\
-c & -d & a & b \\
-d & c & -b & a
\end{array}\right) .
$$


Typically the matrix above represents the quaternion $a+b \mathrm{i}+c \mathrm{j}+d \mathrm{k}$.

Thinking of $i, j, k$ as imaginaries we define a bar conjugation on $\mathbf{H}$ by

$h=\operatorname{Re}(h)+\operatorname{Im}(h) \rightarrow \bar{h}+\operatorname{Re}(h)-\operatorname{Im}(h):$

in terms of the matrix representation above the bar conjugate is just the matrix transpose. So we immediately find that

$\overline{h_{1} h_{2}}=\bar{h}_{2} \bar{h}_{1}$.

We also note that, if $\mathbf{H}$ is thought of as $\mathbf{R}^{+}$with the $1, \mathrm{i}, \mathrm{j} . \mathrm{k}$ basis. the standard norm is just

$\|h\|=(h \bar{h})^{1 / 2}=(\bar{h} h)^{1} 2$.

We extend this to obtain a standard norm on $H "$. defined by

$\| h_{i}=\left(h^{*} h\right)^{1.2}$.

where $h \in \boldsymbol{H}^{n}$ is thought of as a column vector and the asterisk denotes tahing bar conjugate transpose (the multiplication is just matrix multiplication).

There is another notion of conjugation on $\boldsymbol{H}$ defined by

$h^{a}=\alpha^{-1} h \alpha$

where $a \in H$ is nonzero. Note that there are commutation formulas

$$
\begin{aligned}
h_{1} h_{2} & =h_{2} h_{1}^{h}: \\
& =h_{2}^{h_{1}} h_{1} \\
& =h_{2}^{h_{1}} h_{1} .
\end{aligned}
$$

We also define a conjugation on $\boldsymbol{H}$ "in the same spirit by

$h^{n}=\alpha^{-1} h a$

where $\alpha \in \boldsymbol{H}$ is nonzero. $h \in \boldsymbol{H}^{\text {" }}$ and the multiplications are performed component by component.

2.2. By an H-vector space we just mean a right module over $\boldsymbol{H}$.

Example 2.2.1. $\boldsymbol{H}$ is Itself an $\mathbf{H}$-rector space in a natural way. Scalar multiplication is just multiplication on the right. Mvreover. there is an isomorphism $\mathbf{H}-\operatorname{Hom}(\boldsymbol{H}$. H) determined by sending $h$ to multiplication $h$ s $h$, n the left.
Example 2.2.2. The map $H \otimes H \rightarrow H$ defined by $x \otimes a \rightarrow \bar{a} x$

is a right $\boldsymbol{H}$-module structure on $\boldsymbol{H}$. It is isomorphic to the module structure on $H$ in Example 2.2.1 via the bar conjugation map.

Example 2.2.3. $\mathbf{H}^{n}$ is an $\mathbf{H}$-vector space in virtue of being a direct sum of $\boldsymbol{H}$-vector spaces. We continue to think of vectors in $\mathbf{H}^{n}$ as column vectors and find that a linear map $\boldsymbol{A}: \boldsymbol{H}^{m} \rightarrow \boldsymbol{H}^{\prime \prime}$ is just an $m \times n$ matrix of quaternions acting on the left of vectors according to the usual matrix multiplication rules.

Nou, since $\boldsymbol{H}$ is a division algebra. an right $H$-module is a direct sum of copies of $H$ in an essentially unique way. So Example 2.2 .3 gives a good picture of a linear algebra over $\boldsymbol{H}$.

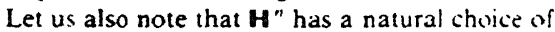
$H$-bimodule structure extending the H-vector space structure of 2.2.3. Consequently. giten an isomorphism of $\mathrm{H}$-rector spaces

$A: M \rightarrow H^{n}$

we can extend the H-vector space structure on $1 /$ to an $\mathbf{H}$-bimodule structure making $A$ an isomor. phism of $\boldsymbol{H}$-bimodules. Linfortunatel thi, itrus: ture is in general dependent in a nontrivial "d! upon the map $A$. This observation essentially explains our choosing to focus attention on module rather than bimodule structures: There are not enough linear maps of $\boldsymbol{H}$-modules. In particular we have:

Proposition 2.2.4. A matrix $A: H^{\prime \prime} \rightarrow H^{\prime \prime}$ presertes the canonical bimadule structures if and onl! if it has real entries.

A somewhat stronger result can be proved as an easy corollary of 2.2.4. It will be useful later.

Corollan 2.2.5. Let $A: H^{\prime \prime} \rightarrow H^{\prime \prime}$. The cummical H-cector space structure on $\mathbf{H}^{\prime \prime}$ extends to an $\mathbf{H - h t}$. module structure which is preserved $h, A$ if and $\mathrm{m} / \mathrm{l}$ if $A$ is conjugate to a real matrix.

\section{Quaternionic linear sy stems}

3.1. In what follous. a system will he a time-invariant linear ordinary differential equation on 


\section{H-vector spaces}

$\dot{x}=A x+B u, \quad x \in \mathbb{Z}, u \in \mathbb{W}$.

Typically we take for granted isomorphisms

$\mathfrak{R} \sim \boldsymbol{H}^{n}, \quad \mathfrak{R}-\boldsymbol{H}^{m}$.

allowing us to think of $A$ and $B$ as matrices of quaternions. We also use the notation $(A . B)$ as a shorthand designation for (3.1.1).

Now the elementary theory of linear O.D.E.'s over $H$ is essentially the same as that of real or complex O.D.E.'s. In particular. we find that there is a well defined matrix exponential

$\mathrm{e}^{t A}=\sum_{n=0}^{\infty} \frac{t^{n}}{n !} A^{n}$.

Moreover, the system (3.1.1) has a unique solution. denoted $x\left(x_{0}, u, t\right)$, which satisfies the initial condition $x\left(x_{0}, u, t\right)=x_{0}$ and which is given by

$x\left(x_{0}, u, t\right)=\mathrm{e}^{t .4} x_{0}+\int_{0}^{t} \mathrm{e}^{(t-s) t} B u(s) \mathrm{d} s$.

Now, the formula just above enables one to prove the following very important theorem.

Theorem 1. Let $M_{1 . B}$ denote the set of states which can be reached by the system $(A, B)$ from the origin in finite time. Then

$\mathbb{R}_{A, B}=\langle A \mid P\rangle=\mathbb{H}+A \mathcal{H}+\cdots+A^{n-1} \mathbb{B}$

where $P$ denotes the image of $B$.

Then. with the usual definition of controllability in mind one can quickly prove:

Theorem 2. The space of matrix pairs

$(A . B) \in \operatorname{Mat}_{n \times n}(H) \times \mathrm{Mat}_{m \times n}(\boldsymbol{H})$.

with $(A, B)$ controllable, is open and dense.

Just as easily. one can use Theorem 1 to prove that controllability is preserved by the action of the feedback groups. that is:

Theorem 3. Change of basis in either state or input space preseries controllability:

Theorem 4. Controllability is preserted by state space feedhack.
3.2. The classical eigenvalue criterion for the stability of a linear system over the real or complex numbers has no clear analogue in the quaternionic case. The reason for this is. in effect. that the classical theory takes liberal advantage of the fact that linear endomorphisms of classical vector spaces preserve the canonical bimodule structure; available.

One way to circumvent this difficulty is to appeal to some real or complex representation of a system whose stability is in question. This approach is considered in [2]. Of course. the control theorist is less interested in testing for stabilits than in stabilizing controllable systems using feedback. So we ought to prove:

Theorem 5. The orbit of any conirollable system. under the action of the feedhack group. coniains a stable sistem.

In fact. we prove Theorem 5 as a corollary of somewhat stronger results in the next section.

\section{Stabilization and invariants}

4.1. The key to stabilizing quaternionic systems is a quaternionic Heymann lemma.

Lemma 4.1.1. Let ( $A . B$ ) be a controlluble sistem over $H$. Then. for any $b$ in the image of $B$. there is $a$ feedback matrix $F$ such that the single-input sistem $(A+B F, b)$ is controllable.

The proof of 4.1 .1 is trivially adapted from the proof of the real Heymann lemma. So we refer the interested reader to [7]. Lemma 3.2.

Given stabilizing feedback for a sistem ( $A+$ $B F, b)$ with $b \in H^{H}$ we can obtain stabilizing feedback for $(A . B)$ by an obvious lifting. So to prote Theorem 5 we need only concern ourselves with the feedback stabilization of single-input systems. We begin by exhibiting a canonical form for controllable single-input systems.

Lemma 4.1.2. Giten a controlluble single-input sy.5tem $(A . b)$ over $H$. there is a unique matrix $A^{\prime}$ of the form 


$$
\left(\begin{array}{ccccc}
0 & 1 & 0 & \cdots & 0 \\
0 & 0 & 1 & & \vdots \\
\vdots & & \ddots & \ddots & 0 \\
0 & \ldots & \cdots & 0 & 1 \\
\alpha_{1} & \alpha_{2} & \cdots & \cdots & \alpha_{n}
\end{array}\right)=A^{\prime}
$$

such that for some $P$ we have

$P^{-1} A P=A^{\prime}$ and $P^{-1} b=e_{n}$

Proof. Let $(A, b)$ be controllable. By Theorem 1 there is a unique $n$-tuple $\alpha_{1} \ldots \ldots \alpha_{n}$ such that

$0=A^{n} b-A^{n-1} b \alpha_{1}-\cdots-b \alpha_{n}$.

Now define a matrix $P=\left[P_{1} \cdots P_{n}\right]$ by the formulas

$P_{1}=A^{n-1} b-A^{n-2} b a_{2} \cdots-b \alpha_{n}$.

$P_{2}=A^{n-2} b-A^{n-3} b a_{3} \cdots-b \alpha_{n}$.

$P_{n-1}=A b-b \alpha_{n} . \quad P_{n}=b$.

Then one easily checks that $A P=P A^{\prime}$ and that $P e_{n}=b$. But by Theorem 1 the $P$, are independent. so $P$ is invertible: and. the proof is complete.

Let us refer to the $\alpha$, in the above as the characteristic indices of the system $(A . b)$ and observe that we have immediately an index assignability theorem.

Theorem 6. The characteristic indices of a controllable single-input sistem can be altered in an arbitran fashion by use of srate-space feedback.

In particular Theorem 6 allow's us to obtain real indices. So Theorem 5 follou's from its real version.

4.2. Recall that in the real Heymann lemma the characteristic indices $a_{1} \ldots \ldots \alpha_{n}$ of the system $(A . b)$ are such that

$\lambda^{n}-\alpha_{1} \lambda^{n-1}-\cdots-\alpha_{n}$

is the characteristic polynomial of $A$. One consequence of this is that the $\alpha$, are independent of the choice of the vector $b$. so long as the system remains controllable.

As it turns out. no such thing occurs in the quaternionic case. It is easy to see uhy:

Suppose the single-input system $(A . b)$ is controllable. Suppose also that at least one characteristic index of $(A, b)$ is nonreal. say $a_{1}$. Then. there are nonzero $\beta \in H$ such that

$\alpha_{1}=\alpha_{1}^{\beta}$.

Now. of course. the system $(A, b \beta)$ is controllable. But. its characteristic indices are $\alpha_{1}^{\beta} \ldots \ldots \alpha_{n}^{\beta}$. To see this just note that by (2.1.1)

$0=A^{n} b \beta-A^{n-1} b \beta \alpha_{1}^{\beta}-\cdots-b \beta a_{n}^{\beta}$.

So by $(4.2 .1),(A, b)$ and $(A, b \beta)$ have distinct characteristic indices.

\section{References}

[1] M. Garcia. J.H. Leuis. and C. Martin. Optumizatuon of large scale symmetric systems. submitted to the $1 /$ th IFIP Conference on System Modeling and Opumization 11983 ).

(2) $M$. Hazewinkel and C. Martin. On decentralizatunn. symme try and special structure in linear systems. suhmitted.

[3] M. Hazeuinkel and C. Martin. Symmetric linear "Ystems: An application of algebraic systems theory. Internat. $J$ Control. to appear.

[4] N. Jacobson. Theon of Rings (Amer. Math. Suc.. Providence. RI. 1943).

[5] C. Martin. Linear decentralized systems with special strus: ture. Internat. J. Control 38 (2) (1982) 291-308

[6] J.H. Lewis and C. Martin. Mathematical models and analy. sis for twinlift helicopter systems. Preprint.

[7] W.M. Wonham. Linear Mulncariable Control: A Cicumetri Approach. Lecture Notes in Economics and Mathematical Systems No. 101 (Springer. Berlin-Neu Yorh. 1974). 